\title{
Transatlantica
}

Revue d'études américaines. American Studies Journal

\section{Le néoconservatisme de Daniel Patrick Moynihan : le cas de la politique étrangère}

Justin Vaïsse

\section{(2) OpenEdition}

1 Journals

Édition électronique

URL : https://journals.openedition.org/transatlantica/4965

DOI : $10.4000 /$ transatlantica.4965

ISSN : $1765-2766$

Éditeur

Association française d'Etudes Américaines (AFEA)

Référence électronique

Justin Vaïsse, "Le néoconservatisme de Daniel Patrick Moynihan : le cas de la politique étrangère », Transatlantica [En ligne], 1 | 2010, mis en ligne le 27 septembre 2010, consulté le 10 février 2023. URL : http://journals.openedition.org/transatlantica/4965; DOI : https://doi.org/10.4000/transatlantica. 4965

Ce document a été généré automatiquement le 10 février 2023.

Creative Commons - Attribution - Pas d'Utilisation Commerciale - Pas de Modification 4.0 International - CC BY-NC-ND 4.0

https://creativecommons.org/licenses/by-nc-nd/4.0/ 


\title{
Le néoconservatisme de Daniel Patrick Moynihan : le cas de la politique étrangère
}

\author{
Justin Vaïsse
}

1 Daniel Patrick Moynihan est une figure archétypale du premier âge du néoconservatisme ${ }^{1}$ : jeune membre de l'ADA (American for Democratic Action) dès les origines, il s'oppose aux tendances pro-communistes (ou du moins de « compagnonnage de route») de Henry Wallace en 1948, et soutient avec enthousiasme le libéralisme du «centre vital $»^{2}$, mélange de progrès social, de keynésianisme et d'anticommunisme. Dans les années 1950, il écrit ses premiers articles dans The Reporter, encouragé par Irving Kristol, père du néoconservatisme, et au milieu des années 1960, il fait partie, avec Daniel Bell ou Norman Podhoretz, des intellectuels qui réagissent contre l'évolution gauchisante du libéralisme américain et "l'ingénierie sociale » des programmes utopiques de la Grande Société, notamment la politique des quotas pour les minorités (cf. la contribution d'Antoine Coppolani).

2 Son parcours est tout aussi emblématique du passage du premier au deuxième âge $d u$ néoconservatisme: les nouveaux arrivants ne s'inquiètent plus seulement de l'évolution du libéralisme, mais directement de celle du parti démocrate. Surtout, la politique étrangère acquiert une place de choix dans leur credo, tandis que leurs aînés se définissaient surtout par rapport aux questions intérieures. Moynihan lui-même avait montré jusque là peu d'intérêt pour les affaires internationales : ne rate-t-il pas le concours de diplomate (FSO) en 1949 ? Ses écrits ont surtout porté sur les questions sociales et politiques - et ce sont elles qui font de lui un néoconservateur, notamment les attaques de « political correctness » avant la lettre de part de la gauche libérale.

Il n'est pourtant pas entièrement étranger aux questions internationales : titulaire d'un $\mathrm{PhD}$ de la Fletcher School of Law and Diplomacy (il représentait l'école à la Student League for World Government, aux idéaux wilsoniens ${ }^{3}$ ), il a poursuivi ses études à la LSE de Londres, et a participé à l'organisation anticommuniste "International Rescue 
Committee » qui aide les réfugiés arrivés aux États-Unis - encore une entreprise marquée au sceau des idéaux américains traditionnels.

Mieux encore, à l'automne 1971, il est nommé délégué américain à l'Assemblée Générale des Nations unies, un poste très peu important mais qui lui fait forte impression. Il y acquiert la conviction que les diplomates américains sont mal armés pour se défendre idéologiquement contre les attaques venues du tiers monde et de l'URSS. Sa volonté de clarté morale dans le domaine international s'affirme : « I will not split the difference between a totalitarian society and an open one, or suggest that there is good to be said on both sides $»^{4}$.

Moynihan est surtout nommé ambassadeur en Inde de février 1973 à début 1975, fonction qu'il doit probablement à sa prise de position originale contre le soutien américain au Pakistan pendant la guerre de 1971, remarquée par Nixon. En Inde, il s'occupe notamment de la "crise de la roupie ", c'est-à-dire la dette énorme que New Delhi devait à Washington ${ }^{5}$. C'est là qu'il acquiert son expérience internationale et se familiarise avec les forces politiques de son temps, en particulier le nationalisme présent dans le tiers monde, et forge sa conviction selon laquelle " tout empire périra " (cf. infra). Il devient à ce moment un critique intransigeant des cleptocraties du Tiers Monde et de leurs apologistes américains... qui sont généralement les libéraux, comme par hasard 6 .

En février 1974, alors qu'il est encore à New Delhi, il publie dans Commentary l'article "Was Woodrow Wilson Right?", et répond par l'affirmative. Dans ce péan à Wilson, il prend comme point de départ ce constat, "We are a nation of nations", et avance que toute lutte pour la liberté et la démocratie dans le monde a forcément un écho aux États-Unis. Il est donc naturel de peser constamment en faveur de la liberté et des régimes démocratiques, tout en sachant le faire avec mesure (pas d'ingérence, pas de « dragons à combattre » notamment). Il conclut en apportant son point de vue original sur les causes de l'antiaméricanisme chez les élites du Tiers monde : la raison en est imputable aux universités britanniques, imprégnées de marxisme, qui ont diffusé cette vulgate nuisible et fausse aux quatre coins de (l'ex-) empire. Il appelle à une fidélité retrouvée aux idéaux de Wilson, à la manière de J.F. Kennedy.

7 Un second article paraît dans Commentary en mars 1975, peu après son retour de New Delhi : dans "The United States in Opposition», cherchant les sources de l'antiaméricanisme tiers-mondiste, il les trouve à nouveau dans les théories socialistes et surtout anticapitalistes britanniques développées entre 1890 et 1950 - qu'il compare aux révolutions américaine, française et soviétique! A en croire Moynihan, les diplomates américains sont aveugles : ils ne reconnaissent pas le poids de l'idéologie en tant que force propre; or la vulgate marxiste, si elle ne fait pas du tiers monde une zone communiste, l'éloigne néanmoins du camp occidental en raison de ses préjugés anticapitalistes et antilibéraux. Moynihan préconise que les États-Unis considèrent désormais l'ONU comme une assemblée parlementaire classique, et adoptent une tactique de minorité, d'opposition, puisque c'est la réalité de l'ordre mondial après la décolonisation. Il faut que le représentant américain se fasse craindre, qu'il n'ait pas peur de dire la vérité, et cesse de s'excuser pour une démocratie imparfaite. Après tout, les régimes qui ont fait passer la liberté avant l'égalité font mieux en termes d'égalité ! Une conférence de presse est organisée pour la publication de son article, ce qui constitue une première dans l'histoire de Commentary. 
8 Cet article est apprécié d'Henry Kissinger, malgré leurs différences de point de vue, et ce dernier lui offre le poste d'ambassadeur aux Nations unies. Moynihan est confirmé en juin, et siège de juillet 1975 à février 1976. Son objectif est clair : conduire une guerre de propagande contre l'antiaméricanisme. À son audition devant le Sénat pour sa confirmation, il déclare : «We are in a propaganda war. We have to respond with a comparable level of effort to that which is directed against us $»^{7}$, en dénonçant les attaques et si besoin en coupant les budgets d'aide aux pays qui critiquent l'Amérique tout en profitant de sa générosité, « [the countries that] have it both ways ». Moynihan dénonce le tiers mondisme, mais critique aussi la Détente ( a form of undisguised retreat »), l'une des marques de fabrique des néoconservateurs dès l'apparition du concept.

9 Une fois ambassadeur, il mène le combat contre la fameuse déclaration de l'Assemblée générale assimilant le sionisme à une forme de racisme, et mène la lutte contre le relativisme moral et l'antiaméricanisme des libéraux et des tiers mondistes. Comme il le dira pendant sa campagne sénatoriale de 1976: "I want to speak up against the charge that we have exploited other countries or that our own prosperity rests on plunder ".

10 Moynihan remporte deux victoires aux Nations unies : d'abord en août, il assure l'échec d'une résolution cubaine à la commission de décolonisation soutenant l'autodétermination et l'indépendance de Puerto Rico. Son travail d'information, soulignant notamment que les Porto-Ricains eux-mêmes avaient déjà rejeté cette option par référendum, s'accompagne de mises en garde aux pays qui seraient tentés par un vote idéologique hostile à l'Amérique : il sera tenu compte de chaque vote - en d'autres termes, la période des «free lunches» est finie. Puis en septembre, à l'Assemblée Générale, sur la question de l'aide publique au développement accordée aux pays du tiers monde, Moynihan propose un texte alternatif de résolution, non marqué par l'antiaméricanisme, et en obtient le passage.

11 Il rencontre aussi des défaites, surtout le 10 novembre 1975, lorsque la résolution "sionisme = racisme " est adoptée. L'ambassadeur Moynihan ne parvient pas à l'empêcher, et doit se contenter d'un discours de dénonciation, certes très brillant et largement médiatisé. Il s'en prend aussi au dictateur Amin Dada après que celui-ci a fait un discours violemment antiaméricain et appelant à l'extinction d'Israël : à San Fransisco, Moynihan le traite de " racist murderer »... et croit bon d'ajouter que ce n'est pas étonnant s'il est chef de l'Organisation de l'Unité Africaine! Ce dernier commentaire déclenche une controverse internationale, au cours de laquelle le Département d'Etat ne soutient pas Moynihan, confirmant celui-ci dans sa vision de Foggy Bottom comme un repaire d' " appeasers ".

12 Il n'en reste pas moins que son opposition brillante à la résolution «sionisme= racisme » lui vaut immense popularité aux États-Unis mêmes, et fait en quelque sorte oublier le «benign neglect memo » du début de la décennie. Il fournit, il est vrai, une sorte de lueur de victoire, au moins rhétorique, à une année 1975 très sombre sur le plan diplomatique ; en d'autres termes, il met du baume au cœur des Américains ${ }^{8}$.

13 Cette soudaine hausse de sa cote de popularité n'est pas étrangère à la détérioration de ses rapports avec Henry Kissinger, lequel ne voit pas d'un bon œil son ambassadeur à l'ONU devenir plus populaire que lui-même. Entre les deux hommes, les irritants se sont accumulés : le 2 juillet 1975, Moynihan n'avait-il pas assisté, avec Scoop Jackson et J. Schlesinger (le Secrétaire à la Défense) au dîner donné par l'AFL-CIO en l'honneur d'A. 
Soljenitsyne, boudé par la Maison blanche et le Département d'Etat? Kissinger multiplie les attaques et les fuites contre Moynihan, par personne interposée; et finalement Moynihan préfère démissionner. Il aura été ambassadeur à l'ONU moins de 8 mois!

Profitant de sa décision de partir, il envoie un câble très critique de la politique étrangère américaine à toutes les ambassades et au Département d'Etat, louant sa propre action à l'ONU, son intransigeance contre l'antiaméricanisme qui paye, contrairement à la mollesse du Département d'Etat, et accusant ce dernier de malveillance à l'égard de la mission à l'ONU. Comme Moynihan avait pris soin de conférer à son télégramme un très bas niveau de classification, celui-ci est vite fuité dans le New York Times... Ford et Kissinger protestent publiquement de leur soutien à l'ambassade américaine à l'ONU, mais en privé l'attaquent durement... il démissionne, comme il l'avait prévu, le 2 février. Au moins cette fois quitte-t-il son poste comme un héros et non comme un paria ${ }^{9}$ !

L'année 1976 est, pour Pat Moynihan, celle des campagnes électorales : il soutient activement Scoop Jackson dans sa campagne des primaires (ce dernier évoquait déjà la possibilité de faire de Moynihan son Secrétaire d'Etat), puis se lance dans sa propre campagne sénatoriale pour l'Etat de New York. On trouve dans son staff de campagne des jeunes néoconservateurs comme Charles Horner et Elliott Abrams, qui viennent de chez Scoop Jackson ${ }^{10}$. Il bat un libéral de gauche, Bella Abzug, de justesse, puis le candidat républicain plus largement.

(Scoop Jackson était, lui, un politicien de longue date), et le meilleur espoir du mouvement, puisque Scoop a perdu à deux reprises. Moynihan continue quelque temps la lutte dans l'orthodoxie néoconservatrice : il critique Paul Warnke, fait l'éloge de la Team B, critique SALT II et toute forme d'appeasement, conduite notamment par Cyrus Vance, de la Rhodésie aux droits de l'Homme (Carter, à ses yeux, « y va doucement avec l'URSS, préférant mettre l'Equateur au pied du mur »).

17 C'est sa période "i icône du néoconservatisme», lorsqu'il est co-président honoraire de la Coalition for a Democratic Majority (CDM) avec Scoop Jackson - c'est par exemple au nom de la CDM qu'il défend, aux côtés de Ben Wattenberg, les idées centristes lors de l'élaboration de la plateforme démocrate de 1976. Il préside de nombreuses réceptions en l'honneur des dissidents cubains ou soviétiques dans les années 77-80, par exemple un dîner de la CDM intitulé « tribute to Sakharov » en février 1980.

Dès 1978, la New Republic évoque une possible candidature présidentielle Moynihan, mais celui-ci va renoncer : " One of the things I know, confie Penn Kemble - venu de la CDM et qui travaille dans son équipe à ce moment - is that when Ted Kennedy announced that he was going to run against Carter, Moynihan said « you have two Irish Catholic from the North East running against Carter, it's gonna be very confusing and tough, people will divide the vote, we'll lose ». Also Kennedy had this incredible machine, a very competent staff and fund-raising capabilities... And Moynihan also felt that if he ran, there would be people in New York who would make him pay a price. Because he would have to run against Carter not only on foreign policy, but also on domestic issues-and in New York, you have that liberal party running people against you, etc. A lot of people thought Moynihan didn't have the temperament-he was too mercurial. "I went to work for him hoping that he would run, but then when he decided not to, I left. $»^{11}$ 
19 Moynihan décide donc de ne pas se présenter aux primaires démocrates, et deux ans après l'élection de Reagan, il déçoit les néoconservateurs en désertant leurs rangs idéologiques.

Il avait déjà montré quelques doutes personnels : ainsi sur le dossier de SALT II, sa position n'était pas tout à fait celle des néoconservateurs, farouchement opposés à la ratification du traité. Eugene Rostow, président du Committee on the Present Danger, lui avait écrit sa déception à ce sujet : «I am disappointed to find you astraddle on the fence. It doesn't suit you, Pat. $»^{12}$

21 Au cours de l'année 1981, il conserve une ligne proche des néoconservateurs sur la plupart des sujets, soutenant par exemple Jeane Kirkpatrick depuis le Sénat (le 2 février 1980, il assiste au déjeuner en l'honneur de sa nomination). En 1982, il se concentre sur les problèmes intérieurs, les plus importants pour sa réélection dans l'État de New York, et prend ses distances vis-à-vis de l'administration Reagan, anathème dans son État. Il n'aime d'ailleurs pas Reagan personnellement, et ne devient pas un «Scoop Jackson » de remplacement au Sénat.

22 Mais c'est surtout après sa réélection qu'il déçoit ses anciens amis - au moment où ceux-ci pensaient qu'il allait revenir vers eux après quelques écarts de campagne électorale bien compréhensibles. En 1983, il fait des déclarations confuses sur l'Amérique centrale, refusant la lecture néoconservatrice de la situation, et critiquant la politique reaganienne. Il devient persuadé que le marxisme-léninisme va s'effondrer partout; il affirmera que dès 1979, il était sûr que l'URSS s'écroulerait sous son propre poids, et reproche aux «neocons » et aux Reaganiens de ne pas voir cette évidence et de s'agiter pour rien. Il ne considère plus le conflit Est-Ouest comme central pour la période historique contemporaine. Ainsi, à la cérémonie de "graduation » de la New York University en 1984, il déclare: "The Soviet idea is spent. It commands some influence in the world; and fear. But it summons no loyalty. History is moving away from it at astounding speed. We must be less obsessed with the Soviets. If we must learn to live with nuclear parity, let us keep all the more in mind that we have consolidated an overwhelming economic advantage." A ses yeux, les vrais problèmes sont le système financier, le terrorisme et le «tribalisme contre la modernité $»^{13}$.

23 A-t-il été prescient ? Un observateur américain au moins n'a jamais tari d'éloge sur les dons prophétiques de Moynihan: c'est Moynihan lui-même... Il a souvent cité son article pour Newsweek en 1979, "Will Russia Blow Up?", notamment dans Pandaemonium en 1993, mais aussi au Sénat en 1991, faisant remonter ses talents de prophète bien avant 1979 :

In these circumstances, in 1979 I wrote an essay for Newsweek-part of a forum on the eighties-in which I said that in the 1980s the Soviet Union would break up and that this could be an intensely dangerous moment for the simple reason that the new insurgent or separatist nations that would emerge could very possibly get hold of nuclear weapons as part of their own defense system against Moscow, or some other hated if nearby capital.[...]

I was raised in New York City and early in life fell among mensheviks, the writers of the New Leader. I listened to Kerenskiy in high school. From the time I was 15, I was given to understand that the Soviet Union would break up and it was only a matter of time. And by the 1970s, I could see that the time was at hand ${ }^{14}$.

Mais ses anciens acolytes se chargent de lui rafraîchir la mémoire : ainsi Penn Kemble, l'un des fondateurs de la CDM, vrai néoconservateur d'origine syndicale, conteste cette vision un peu trop élogieuse, comme bien d'autres ${ }^{15}$. S'il concède qu'en effet, Moynihan 
avait lu attentivement les travaux démographiques de Murray Feshbach et d'autres études de sciences sociales (portant sur l'alcoolisme, les taux de natalité et mortalité, l'espérance de vie en baisse en URSS, etc.) qui pointaient vers un déclin, il rappelle qu'en réalité Moynihan en tirait la conclusion que l'empire soviétique, en déclin mais surarmé, risquait de devenir lui-même plus dangereux, à tel point que son argument des articles de Newsweek était «Be careful of a wounded bear». L'implication, à l'époque, était donc qu'il fallait rester attentifs et forts face à l'URSS - la ligne néoconservatrice en somme! Mais ensuite Moynihan a réinterprété sa position en prétendant qu'il savait qu'il y aurait écroulement, et que c'est pour cela qu'il recommandait de ne pas s'agiter, notamment en Amérique centrale, et de s'inquiéter des suites de l'explosion de l'empire soviétique...

Son abandon des rangs néoconservateurs est marqué notamment par trois propositions de loi de gel des programmes nucléaires («nuclear freeze »), par son opposition au missile MX, et par sa critique virulente de la politique reaganienne en Amérique centrale, y compris en démissionnant pendant un temps de son poste de vice-président de la Commission du renseignement du Sénat pour protester contre le minage des ports du Nicaragua par la CIA et l'invasion de la Grenade.

À travers cet épisode, il marque un attachement de plus en plus grand au respect du droit international ; son wilsonisme devient légaliste, et non plus offensif comme dans les années $1970^{16}$, au grand désarroi de ses amis néoconservateurs qui s'estiment personnellement «trahis ». Midge Decter résume la situation en disant « He broke our hearts »- le néoconservatisme n'est-il pas une affaire de famille? Il est vrai que le mouvement, durement éprouvé dans décennie 1985-1995 par l'extinction de la guerre froide, est orphelin de ses porte-parole : Moynihan le déserte progressivement, Scoop Jackson meurt en 1983, Jeane Kirkpatrick se retire en 1985 et décide finalement de ne pas se présenter à des élections nationales.

Comment expliquer ce tournant "libéral » de Moynihan, lui qui avait été l'icône du néoconservatisme? Il ne s'agit probablement pas que d'une évolution purement intellectuelle et idéologique : les nécessités de la réélection de 1982, dans un contexte où il fallait marquer une séparation nette d'avec l'administration Reagan, jouent sans nul doute un rôle essentiel. Certains évoquent également l'influence de la femme de Moynihan sur son tournant libéral ${ }^{17}$.

De fait, Moynihan devient aussi un défenseur des programmes sociaux qu'il avait attaqués dans les années 1960 et 1970... du moins jusqu'à l'arrivée de Bill Clinton, qui provoque chez lui un réflexe coutumier : s'opposer au nouveau président. Fidèle à sa tradition, il l'attaque donc sur la réforme de l'assurance santé, comme il s'en prendra à la grande réforme de l'aide sociale de 1996 (cf. la contribution de Romain Huret), dernière volte-face d'un intellectuel politicien hors norme, passé du libéralisme mainstream au néoconservatisme orthodoxe, avant d'en revenir à une sorte de néolibéralisme inclassable, où les idiosyncrasies l'emportent sur toute classification politique, sociologique ou intellectuelle. 


\section{NOTES}

1. Sur la succession des trois âges du néoconservatisme, cf. notre article «La croisade des néoconservateurs ", L'Histoire, février 2004.

2. Arthur Schlesinger, The Vital Center, Boston, Houghton Mifflin, 1949.

3. Jacob Heilbrunn, «The Moynihan Enigma ", The American Prospect vol. 8 no.33, July 1, 1997 August 1, 1997.

4. Israel Shenker, " Moynihan Finds UN With Head Lost in Cloud of Ideals ", New York Times, 10 novembre 1971. Cité par John Ehrman, The Rise of Neoconservatism - Intellectuals and Foreign Affairs, 1945 - 1994, Yale University Press, 1995 (ouvrage essentiel dont il est fait un large usage ici).

5. John Ehrman, op. cit.

6. Jacob Heilbrunn, art. cit.

7. Cité par Ehrman, op. cit.

8. John Ehrman, op. cit.

9. John Ehrman, op. cit.

10. Robert G. Kaufman, Henry M. Jackson - A Life in Politics, University of Washington Press, 2000, 337.

11. Entretien avec Penn Kemble, 11 juin 2003, Washington DC.

12. Lettre de Eugene Rostow à Pat Moynihan, 19 novembre 1979, Archives du Committee on the Present Danger, Hoover Institution (Stanford), boîte 207.

13. John Ehrman, op. cit.

14. Congressional Record, Nomination Of Robert M. Gates, Of Virginia, To Be Director Of Central Intelligence (Senate - November 04, 1991) [Page: S15825].

15. Entretien avec Penn Kemble; cf aussi Yahya Sadowski, The Myth of Global Chaos, Brookings Institution Press, 1998, cf. http://brookings.nap.edu/books/0815776640/html/146.html\#pagetop (page consultée le 26 septembre 2010).

16. Jacob Heilbrunn, art. cit.

17. Par exemple Bill Kristol (entretien du 27 juillet 2003, Washington DC).

\section{AUTEUR}

\section{JUSTIN VAIISSE}

Brookings Institution 